\begin{tabular}{|c|c|c|}
\hline 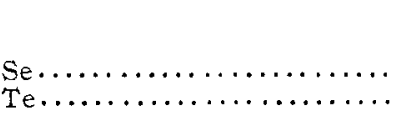 & $\begin{array}{c}\text { I. } \\
\text { Per cent. } \\
0.00251 \\
0.00748\end{array}$ & $\begin{array}{c}\text { II. } \\
\text { Per cent. } \\
0.00449 \\
0.0055^{8}\end{array}$ \\
\hline 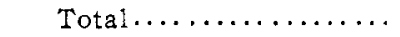 & 0.00999 & 0.01007 \\
\hline
\end{tabular}

The total of the two elements thus calculated, 0.0999 per cent., for I, corresponds closely with the actual amount, 0.0098 per cent., found in the anodes. For II no direct determination was made in the anodes.

BALTIMORE COPPER WORKS, JU1Y, IBg7.

\title{
SOME PRODUCTS OF THE TUBERCULOSIS BACILLUS.
}

BY F. A. DE SCHWEINITZ AND MARION DORSET. Received June :7, $189 \%$.

TBERCULIN, as is well known, is the extract of the tuberculosis bacilli, including the media upon which they are grown. From spenially prepared artificial cultures of the tuberculosis germ, Kühne and independently one of us, ${ }^{1}$ obtained a substance corresponding to a nucleo-albumin, which appears to be the fever-producing principle of the germ. However, many conditions in tuberculosis were not accounted for by this substance, and as Mafucci, Prudden and Hodenpeyl, Vissman, and others, had succeeded in producing tubercular nodules without necrosis by the intravenous injection of dead bacilli, it seemed as though it should be possible to isolate either from cultures or from bodies of the germs themselves, some substance which might be considered accountable for the coagulation necrosis of tissue which takes place, a necrosis which appears necessary for the progress of the disease. This problem was undertaken by my assistant, Dr. Dorset, and myself more than two years ago. After many fruitless attempts we succeeded in isolating from artificial cultures a crystalline substance, having a melting-point of $16 \mathrm{I}^{\circ}-164^{\circ} \mathrm{C}$, readily soluble in ether, alcohol, and water, which separated from these solutions in needle-like or prismatic crystals, showing a slight yellow tint. They did not gire the biuret reaction. The solution of this substance has an acid reaction to litmus, is acid in taste, and is optically inactive. The crystals give no precipitate with silver nitrate, platinic chloride, or harium hydroxide. The analy1 DE Schwernitz; Bulletin No. 7. Bureau of Animal Industry. 
sis showed carbon 50.88 per cent., hydrogen 6.70 per cent., oxygen 43.42 per cent., giving a formula corresponding closely to $\mathrm{C}_{7} \dot{\mathrm{H}}_{10} \mathrm{O}_{4}$. This is the formula of teraconic acid, an unsaturated acid of the fatty series.

The culture media upon which the germs were grown and from which these crystals were obtained, contained potassium acid phosphate, ammonium phosphate, asparagin, or the solution of these salts without asparagin, and glycerol, the medium used by one of us (de Schweinitz) ${ }^{1}$ some years ago for studying these germs. After the germs have been growing on this medium for some weeks the liquid becomes light yellow in color, having the appearance of a pale urine, a change which does not take place in the uninoculated medium kept under the sameconditions. Efforts to obtain this same acid from the ordinary beef broth cultures containing peptone and glycerol resulted in securing minute amounts of the crystals only, which it was never possible to purify. After noting some of the other properties of this acid substance we came to the conclusion that the presence of peptone and the nitrogenous bases of the meat resulted in their combination with the crystals forming compounds from which the acid could not again be easily extracted, even after the addition of acid. Finally, a small quantity of the crystalline substance obtained from the artificial cultures was added to the glycerol peptonized beef broth medium, but it was inpossible to recover it again by the methods used for the first extraction, viz., repeated precipitation with alcohol, solution in water and extraction with ether. The ready solubility of this substance in water, as well as ether, probably accounts for the difficulty of obtaining it. The uninoculated medium did not yield these crystals. When dissolved in water and injected into guinea pigs, this substance caused a reduction of temperature and necrosis of the areas with which it came in contact. As this had been observed in a number of experiments, the idea was suggested that this acid, evidently a secretion of the germ, was one of its most powerful weapons, that by its action upon the tissue the cells were first destroyed so that they could subsequently be utilized by the germ as food, and in this way the germ protected itself from surrounding leucocytes. To test this, crystals dis-

1 New York Medical Journal, 1893 . 
solved in sterile water were injected by means of a hypodermic syringe directly into the liver tissue of a guinea pig. At the same time the same quantity of water was injected into a check in the same way. After forty-eight hours, check and experimental animals were killed. The check failed to show any effect, while the others exhibited a liver with several light spots and necrotic areas. Repetition of this experiment gave the same results.

No effort was made to recover these crystais from the liver as the amount used was too small. As the growth of the tuberculosis bacillus in the body is localized and where localized the necrotic areas are apparent, the fairest test was to bring the substance as soon as possible in contact with the liver tissue.

It seems very reasonable to conclude that we have here the substance formed by the tuberculosis germ, which is responsible for the coagulation necrosis in this disease. The formula which can be deduced from the analysis makes this acid correspond closely to teraconic, which has properties very similar to those noted by us in connection with this new acid. Its identity we have not yet proved or disproved. The amount of this acid obtainable is very small, so that we have used only a very small portion of it for testing its immunizing property. A single injection of 0.0020 gram was sufficient to keep the animais alive some weeks longer than the checks, and its solution appeared to exert some slight bactericidal influence.

As this substance seemed to be a temperature-reacing principle in healthy and diseased animals, we endeavored to separate from tuberculosis cultures the fever-producing principle. The crystals were always found in the culture liquid and only minute amounts could be obtained from the germs themselves that had been grown on liquid media. Accordingly, these germs carefully filtered without heat, were washed with cold water and next extracted with hot water. This hot water extract contained an albuminoid which caused the tuberculin reaction in tuberculous guinea pigs and calves upon repeated injections.

Roux and Nocard claim that they have a tuberculin which will give reactions almost indefinitely, but do not describe its method of preparation. Whether this is the same substance that we have obtained we are unable to say, but certain it is that the tuberculin prepared in the way we have indicated will 
give reactions four or five times in succession where the reaction with tuberculin, as prepared in the ordinary way, fails after the second time. The conclusion is a fair one, we think, that the fever-reducing principle having been removed to an extent, if not entirely, the immunity to the fever-producing principle is much more slowly acquired. In the Deut. Med. Wochen, April I, I 897 , Dr. R. Koch describes some new tuberculin preparations. The dried tuberculosis bacilli were taken (the culture medium used is not mentioned), finely powdered, and centrifugalized with distilled water. The opalescent solution obtained tested upon animals gave the tuberculin reaction. The residual germs were submitted to this treatment a number of times until fnally all were practically dissolved. The latter solutions, he said, in large doses caused a reaction, but in small quantities did not produce this result, and seemed to exert both an immunizing and curative action in experimental tuberculosis. Koch used for this work virulent germs and claims that attenuated germs do not give an active product. Our work was done with germs purposely attenuated by cultivation and the results show that a very active fever-producing, fever-reducing, and probably curative principle can be obtained from them. It hardly seemed justifiable to ourselves to powder dried virulent germs and have the dust floating in the air. Koch further refers to two fatty acids, which, in conjunction with Proskauer, had been found in the bodies of the germs. The writers of this paper published in this Jounnal, August, I 895, a preliminary study of the fats of the tuberculosis bacilli, showing the high content of the fat in the body of these germs, which accounts for the difficulty in staining them with certain colors, as well as their difficult absorption. In a later paper ${ }^{1}$ we described briefly the different acids obtained from these fats, both high-melting and low-melting. Whether these are identical with the acids referred to by Koch we are unable to say, from the brief description he has so far given. This interesting necrotic acid, which plays so important a part apparently in the progress of tuberculosis, and the fever-producing principle formed by the tuberculosis germ are receiving further chemical study. Their physiological importance is very great. The acid may be tentatively called tuberculinic acid.

Brochemic LABoratory, Washington, D. C.

I Centrbl. Bak. u. Parasit., Ig, I8, I9, 707. 